\title{
Singular left-definite Hamiltonian systems in the Sobolev space
}

\author{
Ekin Ugurlu ${ }^{\mathrm{a}}$, Kenan Tas ${ }^{\mathrm{a}}$, Dumitru Baleanu ${ }^{\mathrm{a}, \mathrm{b}, *}$ \\ ${ }^{a}$ Department of Mathematics, Faculty of Arts and Sciences, Cankaya University, 06530 Ankara, Turkey. \\ ${ }^{b}$ Institute of Space Sciences, Magurele-Bucharest, Romania.
}

Communicated by A. Atangana

\begin{abstract}
This paper is devoted to construct Weyl's theory for the singular left-definite even-order Hamiltonian systems in the corresponding Sobolev space. In particular, it is proved that there exist at least $n$-linearly independent solutions in the Sobolev space for the $2 n$-dimensional Hamiltonian system. (C)2017 All rights reserved.
\end{abstract}

Keywords: Hamiltonian system, left-definite problems, Weyl theory. 2010 MSC: 34B20, 46C05.

\section{Introduction}

In 1964, Atkinson studied the following first-order differential equation [1]

$$
J Y^{\prime}=[\lambda A(x)+B(x)] Y, \quad x \in(a, b) \subseteq(-\infty, \infty),
$$

where $J, A$ and $B$ are square matrices of order $k, Y$ is a $k \times 1$ column matrix, $A$ and $B$ are integrable over $(a, b), J$ is a constant matrix and

$$
\mathrm{J}^{*}=-\mathrm{J}, \quad \mathrm{A}^{*}(\mathrm{x})=\mathrm{A}(\mathrm{x}) \geqslant 0, \quad \mathrm{~B}^{*}(\mathrm{x})=\mathrm{B}(\mathrm{x}) .
$$

Equation (1.1) is called the Hamiltonian system and contains kth order formally selfadjoint differential equations [23] as well as more interesting differential equations.

Equation (1.1) with conditions (1.2), especially with $A(x) \geqslant 0$, has been studied for right-definite equations in the Hilbert space $L_{A}^{2}(a, b)$ which is equipped with the inner product

$$
(Y, Z)=\int_{a}^{b} Z^{*} A Y d x
$$

\footnotetext{
${ }^{*}$ Corresponding author

Email addresses: ekinugurlu@cankaya.edu.tr (Ekin Ugurlu), kenan@cankaya.edu.tr (Kenan Tas), dumitru@cankaya.edu.tr (Dumitru Baleanu)

doi:10.22436/jnsa.010.08.37
} 
In particular, in 1910, Weyl proved with his extraordinary way the second-order equation

$$
-\left(p(x) y^{\prime}\right)^{\prime}+q(x) y=\lambda y, \quad x \in[0, \infty),
$$

has at least one solution $\chi(x, \lambda)=\varphi(x, \lambda)+m_{\infty}(\lambda) \psi(x, \lambda)$ satisfying

$$
\int_{a}^{\infty}|x|^{2} \mathrm{~d} x<\infty
$$

where $p$ and $q$ are real-valued functions on the given interval, $\varphi$ and $\psi$ are linearly independent solutions of (1.3) and $m_{\infty}$ is a point on the limiting-point or limiting-circle [25]. Atkinson generalized this result for the linear $2 n$-dimensional Hamiltonian system (1.1) satisfying (1.2) and he proved that at least n-linearly independent solutions of (1.1), (1.2) lie in $\mathrm{L}_{A}^{2}(a, b)$.

A different approach was given by Niessen [17-19]. Niessen examined the matrix

$$
\mathcal{A}(x)=(1 / 2 \operatorname{Im} \lambda) y^{*}(x, \lambda)(J / i) y(x, \lambda), \quad \operatorname{Im} \lambda \neq 0,
$$

where $y(x, \lambda)$ is the fundamental solution of (1.1), (1.2). However, more efficient method was introduced by Hinton and Shaw [4-6]. In this method, Hinton and Shaw used matrix function $M(\lambda)$ which is similar with Weyl's function to construct the circle or ellipsoid equations. Then they proved that (1.1), (1.2) have at least $n$-linearly independent solutions belonging to $L_{A}^{2}(a, b)$. Similar approach was given by Krall $[8,12]$.

However, all these results were introduced for the right-definite Hamiltonian systems. Right-definite case is related with the right-hand side of (1.1). Positiveness condition in the right-hand side of the equation generates a weighted Hilbert space. On the other side, in real-world problems there exist functions in the right-hand side of the equations changing the sign on the interval. As a famous application we can give the Camassa-Holm equation [2]

$$
-y^{\prime \prime}+\frac{1}{4} y=\lambda w(x) y
$$

To introduce the motivation of the left-definite equations consider the equation

$$
y^{\prime \prime}+y=0 .
$$

Multiplying (1.4) with $y^{\prime}$, it is found that

$$
\left(y^{\prime}\right)^{2}+y^{2}=c^{2}
$$

where $c$ is a constant. Solving for $y^{\prime}$ we have

$$
y^{\prime}=\sqrt{c^{2}-y^{2}}
$$

Choosing $y=c_{1} \sin \gamma$ it is obtained

$$
y=c_{1} \sin \left(x+c_{2}\right),
$$

where $c_{1}, c_{2}$ are constants. The left-side of (1.5) may arise in the standart Sturm-Liouville equations. In fact, for sufficiently nice functions one obtains

$$
\int_{c}^{d}\left[-\left(p y^{\prime}\right)^{\prime}+q y\right] \bar{y} d x=\int_{c}^{d}\left[p\left|y^{\prime}\right|^{2}+q|y|^{2}\right] d x-\left.\left(p y^{\prime}\right) \bar{y}\right|_{c} ^{d},
$$

where $-\infty \leqslant c<d \leqslant \infty$. Therefore imposing positiveness condition on $p$ and $q$ one can construct the Sobolev space $\mathrm{H}^{1}(\mathrm{c}, \mathrm{d} ; \mathrm{p}, \mathrm{q})$ with the inner product

$$
\langle y, z\rangle=\int_{c}^{d}\left[p y^{\prime} \bar{z}^{\prime}+q y \bar{z}\right] d x .
$$


In recent years, the authors have studied some spectral properties of the regular and singular left-definite Sturm-Liouville differential and difference equations [2, 3, 7, 14-16, 20, 24].

In 1995, Krall and Race [13] studied the singular left-definite second-order Sturm-Liouville equation

$$
-\left(p y^{\prime}\right)^{\prime}+q y=\lambda w y, \quad(a, b) \subseteq(-\infty, \infty),
$$

where $p, q, w$ are real-valued, positive functions over $(a, b)$ such that $p^{-1}$ is locally integrable on $(a, b)$, $\epsilon_{1} w \leqslant q \leqslant \epsilon_{2} w, q$ and $w$ are in $L^{1}(a, b)$ (also see $[9,10]$ ). They proved that there is a solution $\chi(x, \lambda)=\varphi(x, \lambda)+m_{b}(\lambda) \psi(x, \lambda)$ of (1.6) belonging to $H^{1}(a, b ; p, q)$. However, for a singular left-definite fourth/sixth/... order or a linear singular left-definite Hamiltonian system has not been studied yet. Beside this, Weyl's theory for the singular Dirac system has been investigated in [21]. In this paper, our main aim is to develop Weyl's theory for the singular left-definite linear even-dimensional Hamiltonian system. It should be noted that regular left-definite Hamiltonian system has been studied in [11] and some properties of the regular fractional operator in the Sobolev space has been investigated in [22].

\section{Preliminaries}

In this section we shall remind some known results on singular right-definite Hamiltonian system in $\mathrm{L}_{A}^{2}(\mathrm{a}, \mathrm{b})$.

Let us assume that $a$ is the regular point and $b$ is the singular point for the $2 n$-dimensional Hamiltonian system (1.1), (1.2). Let $y$ be a fundamental matrix of size $2 n \times 2 n$ of (1.1), (1.2) satisfying

$$
y(a, \lambda)=\left(\begin{array}{ll}
\alpha_{1}^{*} & -\alpha_{2}^{*} \\
\alpha_{2}^{*} & \alpha_{1}^{*}
\end{array}\right),
$$

where $\alpha_{1}, \alpha_{2}$ are $n \times n$ real-matrices such that $\operatorname{rank}\left(\alpha_{1}, \alpha_{2}\right)=n$,

$$
\alpha_{1} \alpha_{1}^{*}+\alpha_{2} \alpha_{2}^{*}=I_{n}, \quad \alpha_{1} \alpha_{2}^{*}-\alpha_{2} \alpha_{1}^{*}=0,
$$

and $I_{n}$ is the $n \times n$ identity matrix. If $y$ is partitioned into

$$
Y=\left(\begin{array}{ll}
\theta & \phi
\end{array}\right)=\left(\begin{array}{ll}
\theta_{1} & \phi_{1} \\
\theta_{2} & \phi_{2}
\end{array}\right)
$$

we may assume that

$$
\left(\begin{array}{ll}
\alpha_{1} & \alpha_{2}
\end{array}\right) \theta(a)=I_{n}, \quad\left(\begin{array}{ll}
\alpha_{1} & \alpha_{2}
\end{array}\right) \phi(a)=0 .
$$

Now consider the following boundary condition $a t b^{\prime}, b^{\prime}<b$,

$$
\left(\begin{array}{ll}
\beta_{1} & \beta_{2}
\end{array}\right) Y\left(b^{\prime}\right)=0,
$$

where $\beta_{1}, \beta_{2}$ are $n \times n$ real-matrices such that $\operatorname{rank}\left(\beta_{1}, \beta_{2}\right)=n$ and

$$
\beta_{1} \beta_{1}^{*}+\beta_{2} \beta_{2}^{*}=I_{n}, \quad \beta_{1} \beta_{2}^{*}-\beta_{2} \beta_{1}^{*}=0 .
$$

We set the solution $\chi$ of (1.1), (1.2) as

$$
x=y\left(\begin{array}{l}
I_{n} \\
M\left(b^{\prime}\right)
\end{array}\right) .
$$

Then $\chi$ satisfies the boundary condition (2.1) at $b^{\prime}$ if

$$
M\left(b^{\prime}\right)=-\left(\beta_{1} \phi_{1}\left(b^{\prime}, \lambda\right)+\beta_{2} \phi_{2}\left(b^{\prime}, \lambda\right)\right)^{-1}\left(\beta_{1} \theta_{1}\left(b^{\prime}, \lambda\right)+\beta_{2} \theta_{2}\left(b^{\prime}, \lambda\right)\right),
$$

and $\chi^{*}\left(b^{\prime}, \lambda\right) J \chi\left(b^{\prime}, \lambda\right)=0$, where 


$$
J=\left(\begin{array}{ll}
0 & -I_{n} \\
I_{n} & 0
\end{array}\right)
$$

Circle equation can be introduced as

$$
\pm\left(\begin{array}{ll}
I_{n} & M^{*}
\end{array}\right) y^{*}\left(b^{\prime}\right)(J / i) y\left(b^{\prime}\right)\left(\begin{array}{c}
I_{n} \\
M
\end{array}\right)=0
$$

where " + " holds when $\operatorname{Im} \lambda>0$ and "- " holds when $\operatorname{Im} \lambda<0$.

Now let

$$
\left(\begin{array}{ll}
\mathcal{A} & \mathcal{B}^{*} \\
\mathcal{B} & \mathcal{D}
\end{array}\right)=\left\{\begin{array}{l}
y^{*}\left(b^{\prime}\right)(J / \mathfrak{i}) y\left(b^{\prime}\right), \quad \operatorname{Im} \lambda>0 \\
-y^{*}\left(b^{\prime}\right)(J / i) y\left(b^{\prime}\right), \quad \operatorname{Im} \lambda<0
\end{array}\right.
$$

and

$$
\mathbb{E}(M)=\left(\begin{array}{ll}
I_{n} & M^{*}
\end{array}\right)\left(\begin{array}{ll}
\mathcal{A} & \mathcal{B}^{*} \\
\mathcal{B} & \mathcal{D}
\end{array}\right)\left(\begin{array}{l}
I_{n} \\
M
\end{array}\right)
$$

The circle equation $\mathbb{E}(M)=0$ can be expressed as

$$
\mathbb{E}(M)=(M-C)^{*} R_{1}^{-2}(M-C)-R_{2}^{2}=0,
$$

where $C=-\mathcal{D}^{-1} \mathcal{B}, R_{1}=\mathcal{D}^{-1 / 2}, R_{2}=\left(\mathcal{B}^{*} \mathcal{D}^{-1} \mathcal{B}-\mathcal{A}\right)^{1 / 2}$.

Then following theorem is valid $[8,12]$.

\section{Theorem 2.1.}

(i) $\mathcal{D}>0$;

(ii) $\mathcal{B}^{*} \mathcal{D}^{-1} \mathcal{B}-\mathcal{A}=\mathcal{D}^{-1}(\bar{\lambda})$;

(iii) $\mathrm{R}_{2}=\overline{\mathrm{R}}_{1}$;

(iv) as $\mathrm{b}^{\prime}$ increases, $\mathcal{D}$ increases, $\mathrm{R}_{1}$ decreases and $\mathrm{R}_{2}$ decreases;

(v) $\lim _{\mathrm{b}^{\prime} \rightarrow \mathrm{b}} \mathrm{R}_{1}\left(\mathrm{~b}^{\prime}, \lambda\right)=\mathrm{R}_{0}(\lambda)=\mathrm{R}_{0}, \lim _{\mathrm{b}^{\prime} \rightarrow \mathrm{b}} \mathrm{R}_{2}\left(\mathrm{~b}^{\prime}, \lambda\right)=\mathrm{R}_{0}(\bar{\lambda})=\widetilde{\mathrm{R}}_{0}, \mathrm{R}_{0} \geqslant 0, \widetilde{R}_{0} \geqslant 0$;

(vi) as $\mathrm{b}^{\prime}$ approaches $\mathrm{b}$, the circles $\mathbb{E}(\mathrm{M})=0$ are nested and $\lim _{\mathrm{b}^{\prime} \rightarrow \mathrm{b}} \mathrm{C}\left(\mathrm{b}^{\prime}, \lambda\right)=\mathrm{C}_{0}$ exists;

(vii) $\mathrm{M}=\mathrm{C}_{0}+\mathrm{R}_{0} \mathrm{U} \overline{\mathrm{R}}_{0}, \mathrm{U}=\mathrm{R}_{1}^{-1}(\mathrm{M}-\mathrm{C}) \overline{\mathrm{R}}_{1}^{-1}$ is well-defined. As $\mathrm{U}$ varies over the unit-circle in the $\mathrm{n} \times \mathrm{n}$ sphere the limit-circle or -point $\mathbb{E}_{0}(M)$ is covered.

\section{Dirichlet formula}

To construct the Sobolev space we let [11]

$$
A=\left(\begin{array}{ll}
E & 0 \\
0 & 0
\end{array}\right), B=\left(\begin{array}{ll}
-B_{11} & B_{12} \\
B_{12}^{*} & B_{22}
\end{array}\right),
$$

and $-B_{11} \leqslant 0 \leqslant B_{22}, \rho E \leqslant B_{11}$. Therefore classical $L_{A}^{2}(a, b)$ space implies

$$
(Y, Z)=\int_{a}^{b} Z^{*} A Y d x=\int_{a}^{b} Z_{1}^{*} E Y_{1} d x .
$$

On the other hand, Sobolev space $\mathrm{H}^{1}\left(\mathrm{a}, \mathrm{b} ; \mathrm{B}_{11}, \mathrm{~B}_{22}\right)$ is equipped with the inner product

$$
\langle Y, Z\rangle=\int_{a}^{b} Z^{*}\left(\begin{array}{ll}
B_{11} & 0 \\
0 & B_{22}
\end{array}\right) Y d x=\int_{a}^{b}\left[Z_{1}^{*} B_{11} Y_{1}+Z_{2}^{*} B_{22} Y_{2}\right] d x
$$


Now consider the equations

$$
J Y^{\prime}-B Y=A F, \quad L Y:=F,
$$

where $Y, F \in L_{A}^{2}(a, b)$. Then

$$
\begin{aligned}
& -Y_{2}^{\prime}+B_{11} Y_{1}-B_{12} Y_{2}=E F_{1} \\
& Y_{1}^{\prime}-B_{12}^{*} Y_{1}-B_{22} Y_{2}=0 .
\end{aligned}
$$

Therefore

$$
\begin{aligned}
(L Y, Z) & =\int_{a}^{b} Z_{1}^{*}\left[-Y_{2}^{\prime}+B_{11} Y_{1}-B_{12} Y_{2}\right] d x \\
& =-\left.Z_{1}^{*} Y_{2}\right|_{a} ^{b}+\int_{a}^{b}\left[Z_{1}^{*^{\prime}} Y_{2}+Z_{1}^{*} B_{11} Y_{1}-Z_{1}^{*} B_{12} Y_{2}\right] d x \\
& =-\left.Z_{1}^{*} Y_{2}\right|_{a} ^{b}+\int_{a}^{b}\left[\left(B_{12}^{*} Z_{1}+B_{22} Z_{2}\right)^{*} Y_{2}+Z_{1}^{*} B_{11} Y_{1}-Z_{1}^{*} B_{12} Y_{2}\right] d x \\
& =-\left.Z_{1}^{*} Y_{2}\right|_{a} ^{b}+\int_{a}^{b}\left[Z_{1}^{*} B_{11} Y_{1}+Z_{2}^{*} B_{22} Y_{2}\right] d x,
\end{aligned}
$$

provided that

$$
\mathrm{Z}_{1}^{\prime}-\mathrm{B}_{12}^{*} \mathrm{Z}_{1}=\mathrm{B}_{22} \mathrm{Z}_{2}
$$

Hence we have the Dirichlet formula

$$
(\mathrm{LY}, \mathrm{Z})=\langle\mathrm{Y}, \mathrm{Z}\rangle-\left.\mathrm{Z}_{1}^{*} \mathrm{Y}_{2}\right|_{\mathrm{a}} ^{\mathrm{b}}
$$

\section{Singular left-definite Hamiltonian system}

In this section we introduce the main results.

Equation (3.2) implies that

$$
\lambda \int_{a}^{b^{\prime}} Y_{1}^{*} E_{1} Y_{1} d x=\int_{a}^{b^{\prime}} Y_{1}^{*} B_{11} Y_{1} d x+\int_{a}^{b^{\prime}} Y_{2}^{*} B_{22} Y_{2} d x-\left.Y_{1}^{*} Y_{2}\right|_{a} ^{b^{\prime}}
$$

Now consider the boundary condition (2.1) at $b^{\prime}$. Then the solution

$$
x=y\left(\begin{array}{l}
I_{n} \\
M\left(b^{\prime}\right)
\end{array}\right),
$$

satisfies (2.1) if $M\left(b^{\prime}\right)$ is of the form (2.2). Equation (4.1) implies that

$$
\begin{aligned}
\lambda \int_{a}^{b^{\prime}} \chi_{1}^{*} E \chi_{1} d x & =\int_{a}^{b^{\prime}} \chi_{1}^{*} B_{11} \chi_{1} d x+\int_{a}^{b^{\prime}} \chi_{2}^{*} B_{22} \chi_{2} d x-\left.\chi_{1}^{*} \chi_{2}\right|_{a} ^{b^{\prime}} \\
& =\int_{a}^{b^{\prime}} \chi_{1}^{*} B_{11} \chi_{1} d x+\int_{a}^{b^{\prime}} \chi_{2}^{*} B_{22} \chi_{2} d x-\chi_{1}^{*}\left(b^{\prime}\right) \chi_{2}\left(b^{\prime}\right)+\chi_{1}^{*}(a) \chi_{2}(a) .
\end{aligned}
$$

Note that

$$
\left(\begin{array}{l}
\chi_{1}(a) \\
\chi_{2}(a)
\end{array}\right)=\left(\begin{array}{l}
\alpha_{1}^{*}-\alpha_{2}^{*} M\left(b^{\prime}\right) \\
\alpha_{2}^{*}+\alpha_{1}^{*} M\left(b^{\prime}\right)
\end{array}\right),\left(\begin{array}{l}
\chi_{1}\left(b^{\prime}\right) \\
\chi_{2}\left(b^{\prime}\right)
\end{array}\right)=\left(\begin{array}{l}
\beta_{2}^{*} \\
-\beta_{1}^{*}
\end{array}\right)
$$


Substitution (4.3) into (4.2) we find

$$
\begin{aligned}
\lambda \int_{a}^{b^{\prime}} \chi_{1}^{*} E \chi_{1} d x= & \int_{a}^{b^{\prime}} \chi_{1}^{*} B_{11} \chi_{1} d x+\int_{a}^{b^{\prime}} \chi_{2}^{*} B_{22} \chi_{2} d x+\beta_{2} \beta_{1}^{*}-M^{*} \alpha_{2} \alpha_{2}^{*} \\
& -M^{*} \alpha_{2} \alpha_{1}^{*} M+\alpha_{1} \alpha_{2}^{*}+\alpha_{1} \alpha_{1}^{*} M .
\end{aligned}
$$

Now, let $\beta_{1}=0$ such that $\operatorname{rank} \beta_{2}=n$. This case corresponds to

$$
\chi_{2}\left(b^{\prime}\right)=0,
$$

and (2.2) coincides with

$$
M\left(b^{\prime}\right)=-\phi_{2}^{-1}\left(b^{\prime}\right) \theta_{2}\left(b^{\prime}\right) .
$$

Then (4.4) gives

$$
\begin{aligned}
\int_{a}^{b^{\prime}} \chi_{1}^{*} B_{11} \chi_{1} d x+\int_{a}^{b^{\prime}} \chi_{2}^{*} B_{22} \chi_{2} d x= & \lambda \int_{a}^{b^{\prime}} \chi_{1}^{*} E x_{1} d x+M^{*} \alpha_{2} \alpha_{2}^{*} \\
& +M^{*} \alpha_{2} \alpha_{1}^{*} M-\alpha_{1} \alpha_{2}^{*}-\alpha_{1} \alpha_{1}^{*} M .
\end{aligned}
$$

Fixing $b^{\prime}$ in the upper limit in the integral we find

$$
\begin{aligned}
\int_{a}^{b^{\prime}} \chi_{1}^{*} B_{11} \chi_{1} d x+\int_{a}^{b^{\prime}} \chi_{2}^{*} B_{22} \chi_{2} d x \leqslant & \operatorname{Re} \lambda \int_{a}^{b} \chi_{1}^{*} E \chi_{1} d x-\alpha_{1} \alpha_{2}^{*}+\operatorname{Re}\left\{M^{*}(b) \alpha_{2} \alpha_{2}^{*}\right. \\
& \left.+M^{*}(b) \alpha_{2} \alpha_{1}^{*} M(b)-\alpha_{1} \alpha_{1}^{*} M(b)\right\}
\end{aligned}
$$

This implies the following theorem.

Theorem 4.1. There exists a solution

$$
x=y\left(\begin{array}{l}
I_{n} \\
M(b)
\end{array}\right)
$$

of (1.1), (3.1) such that for all $\lambda$ with $\operatorname{Im} \lambda \neq 0$ lies in $\mathrm{H}^{1}\left(\mathrm{a}, \mathrm{b} ; \mathrm{B}_{11}, \mathrm{~B}_{22}\right)$.

Remark 4.2. It seems that there is no need to consider $\beta_{1}=0$ to introduce Theorem 4.1. However, in [13] Krall and Race showed that there is needed to restrict the boundary conditions for further calculation in their work. So we take $\beta_{1}=0$ to coincide the further results in [13].

Theorem 4.3. Let $\operatorname{rank} R_{0}=r_{1}, \operatorname{rank} \bar{R}_{0}=r_{2}, v=n+\min \left(r_{1}, r_{2}\right)$. Then for $\operatorname{Im} \lambda \neq 0$, there exist $v$ solutions of (1.1) satisfying

$$
\int_{\mathbf{a}}^{\mathbf{b}^{\prime}} Y_{1}^{*} B_{11} Y_{1} d x+\int_{\mathbf{a}}^{\mathbf{b}^{\prime}} Y_{2}^{*} B_{22} Y_{2} d x<\infty
$$

Proof. Consider the solution

$$
\chi(x, \lambda)=y(x, \lambda)\left(\begin{array}{l}
I_{n} \\
C_{0}
\end{array}\right)=\left(\begin{array}{lll}
Y_{1} & \cdots & Y_{n}
\end{array}\right)(x, \lambda) .
$$

Then

$$
\int_{a}^{b^{\prime}} Y_{j, 1}^{*} B_{11} Y_{j, 1} d x+\int_{a}^{b^{\prime}} Y_{j, 2}^{*} B_{22} Y_{j, 2} d x<\infty,
$$


where $Y_{j}, 1 \leqslant j \leqslant n$, are $n$-linearly independent solutions.

Now let

$$
\tilde{x}(x, \lambda)=y(x, \lambda)\left(\begin{array}{l}
I_{n} \\
M(b)
\end{array}\right)=\left(\begin{array}{lll}
Z_{1} & \cdots & Z_{n}
\end{array}\right)(x, \lambda),
$$

where $M(b)=C_{0}+R_{0} U \bar{R}_{0}$ and $U^{*} U \leqslant I_{n}$. Therefore

$$
\int_{a}^{b^{\prime}} Z_{k, 1}^{*} B_{11} Z_{k, 1} d x+\int_{a}^{b^{\prime}} Z_{k, 2}^{*} B_{22} Z_{k, 2} d x<\infty,
$$

where $Z_{j}, n+1 \leqslant k \leqslant 2 n$, are $n$-linearly independent solutions. Therefore

$$
\left(\begin{array}{llllll}
Y_{1} & \cdots & Y_{n} & Z_{1} & \cdots & Z_{n}
\end{array}\right)(x, \lambda)=y(x, \lambda)\left(\begin{array}{ll}
I_{n} & I_{n} \\
C_{0} & M(b)
\end{array}\right) .
$$

One can write

$$
\left(\begin{array}{ll}
I_{n} & I_{n} \\
C_{0} & M(b)
\end{array}\right)=\left(\begin{array}{ll}
I_{n} & 0 \\
C_{0} & R_{0} U \bar{R}_{0}
\end{array}\right)\left(\begin{array}{ll}
I_{n} & I_{n} \\
0 & I_{n}
\end{array}\right) .
$$

Equation (4.5) shows that

$$
\operatorname{rank}\left(\begin{array}{ll}
I_{n} & I_{n} \\
C_{0} & M(b)
\end{array}\right)=n+\min \left(r_{1}, r_{2}\right)=v .
$$

Since the right matrix on the right side in (4.5) and $y(x, \lambda)$ are invertible,

$$
\operatorname{rank}\left(\begin{array}{llllll}
Y_{1} & \cdots & Y_{n} & Z_{1} & \cdots & Z_{n}
\end{array}\right)=v,
$$

and this completes the proof.

\section{References}

[1] F. V. Atkinson, Discrete and continuous boundary problems, Mathematics in Science and Engineering, Academic Press, New York-London, (1964). 1

[2] C. Bennewitz, B. M. Brown, R. Weikard, Inverse spectral and scattering theory for the half-line left-definite SturmLiouville problem, SIAM J. Math. Anal., 40 (2009), 2105-2131. 1, 1

[3] A. Bruder, L. L. Littlejohn, Classical and Sobolev orthogonality of the nonclassical Jacobi polynomials with parameters $\alpha=\beta=-1$, Ann. Mat. Pura Appl., 193 (2014), 431-455. 1

[4] D. B. Hinton, J. K. Shaw, On Titchmarsh-Weyl M( $\lambda)$-functions for linear Hamiltonian systems, J. Differential Equations, 40 (1981), 316-342. 1

[5] D. Hinton, K. Shaw, Titchmarsh-Weyl theory for Hamiltonian systems, Spectral theory of differential operators, Birmingham, Ala., (1981), North-Holland Math. Stud., North-Holland, Amsterdam-New York, 55 (1981), $219-231$.

[6] D. B. Hinton, J. K. Shaw, Parameterization of the $M(\lambda)$ function for a Hamiltonian system of limit circle type, Proc. Roy. Soc. Edinburgh Sect. A, 93 (1983), 349-360. 1

[7] Q. Kong, H. Wu, A. Zettl, Left-definite Sturm-Liouville problems, J. Differential Equations, 177 (2001), 1-26. 1

[8] A. M. Krall, $\mathrm{M}(\lambda)$ theory for singular Hamiltonian systems with one singular point, SIAM J. Math. Anal., 20 (1989), 664-700. 1, 2

[9] A. M. Krall, $\mathrm{H}^{1}$ Bessel expansions, Indian J. Pure Appl. Math., 26 (1995), 51-61. 1

[10] A. M. Krall, $\mathrm{H}^{1}$ convergence of Fourier integrals, Indian J. Pure Appl. Math., 26 (1995), 41-50. 1

[11] A. M. Krall, Left-definite regular Hamiltonian systems, Math. Nachr., 174 (1995), 203-217. 1, 3

[12] A. M. Krall, Hilbert space, boundary value problems and orthogonal polynomials, Operator Theory: Advances and Applications, Birkhäuser Verlag, Basel, (2002). 1, 2

[13] A. M. Krall, D. Race, Self-adjointness for the Weyl problem under an energy norm, Quaestiones Math., 18 (1995), 407-426. 1, 4.2

[14] C. Liaw, L. L. Littlejohn, J. Stewart, Q. Wicks, A spectral study of the second-order exceptional $\mathrm{X}_{1}$-Jacobi differential expression and a related non-classical Jacobi differential expression, J. Math. Anal. Appl., 422 (2015), 212-239. 1

[15] L. L. Littlejohn, R. Wellman, On the spectra of left-definite operators, Complex Anal. Oper. Theory, 7 (2013), $437-455$.

[16] L. L. Littlejohn, Q. Wicks, Glazman-Krein-Naimark theory, left-definite theory and the square of the Legendre polynomials differential operator, J. Math. Anal. Appl., 444 (2016), 1-24. 1

[17] H. D. Niessen, Singuläre S-Hermitesche Rand-Eigenwertprobleme, Manuscripta Math., 3 (1970), 35-68. 1 
[18] H. D. Niessen, Zum verallgemeinerten zweiten Weylschen Satz, (German) Arch. Math. (Basel), 22 (1971), $648-656$.

[19] H. D. Niessen, Greensche Matrix und die Formel von Titchmarsh-Kodaira für singuläre S-hermitesche Eigenwertprobleme, (German) J. Reine Angew. Math., 261 (1973), 164-193. 1

[20] G.-L. Shi, R. Yan, Spectral theory of left definite difference operators, J. Math. Anal. Appl., 337 (2008), 116-122. 1

[21] E. Uğurlu, Singular Dirac systems in the Sobolev space, Turk. J. Math., 41 (2017), 933-939. 1

[22] E. Uğurlu, D. Baleanu, K. Tas, Regular fractional differential equations in the Sobolev space, Fract. Calc. Appl. Anal., 20 (2017), 810-817. 1

[23] P. W. Walker, A vector-matrix formulation for formally symmetric ordinary differential equations with applications to solutions of integrable square, J. London Math. Soc., 9 (1974), 151-159. 1

[24] G.-S. Wei, S.-Z. Fu, Left-definite spaces of singular Sturm-Liouville problems, J. Math. Anal. Appl., 345 (2008), $420-430$. 1

[25] H. Weyl, Über gewöhnliche Differentialgleichungen mit Singularitäten und die zugehörigen Entwicklungen willkürlicher Funktionen, (German) Math. Ann., 68 (1910), 220-269. 1 